\title{
Comunicación educativa, educomunicación y educación mediática: una propuesta de investigación y formación desde un enfoque culturalista ${ }^{\star}$
}

\section{Ancízar Narváez-Montoya ${ }^{1}$}

Recibido: 27/03/2017

Aprobado por pares: 09/04/2018
Enviado a pares: $02 / 03 / 2018$

Aceptado: 09/04/2018

DOI: 10.5294/pacla.2019.22.3.11

Para citar este artículo / to reference this article / para citar este artigo

Narváez-Montoya, A. (2019). Comunicación educativa, educomunicación y educación mediática: una propuesta de investigación y formación desde un enfoque culturalista. Palabra Clave, 22(3), e22311. DOI: http://doi.org/10.5294/pacla.2019.22.3.11

\section{Resumen}

Entre educación presencial y virtual, escuela y medios e interacción e interactividad, se suele debatir el campo de la educomunicación. Por otro lado, esta se ha entendido como educación sobre los medios o por los medios, por lo cual siempre se ha tendido a confundir con educación mediática. El objetivo de este artículo es hacer una revisión del concepto básico de comunicación educativa y sus derivaciones: educomunicación y educación mediática, para luego redefinirlos desde un punto de vista culturalista. Desde este enfoque, se aplican las categorías de la teoría de los códigos a la llamada educomunicación, que da como resultado la existencia de tres tipos de educomunicación: oral-ritual, icónico-narrativa y alfabético-argumentativa (paradigmática). En conclusión, se propone la educomunicación como la totalidad de la subjetivación humana, entendida como la adquisición de

\footnotetext{
* Este artículo hace parte de los antecedentes y de la elaboración teórica del proyecto Educación mediática y nación: entre el púlpito e internet, actualmente en desarrollo bajo el código DPG-437-16 del Centro de Investigaciones de la Universidad Pedagógica Nacional y coordinado por el autor.

1 orcid.org/0000-0003-1429-3935. Universidad Pedagógica Nacional de Colombia, Colombia. anarvaez@pedagogica.edu.co
} 
diferentes códigos, entre ellos, la lengua particular, yla educación mediática como la asunción de los códigos mediáticos, entendidos no como tecnologías sino como cultura. El aporte fundamental es la ampliación del concepto educomunicación al incorporar, además del análisis de las tecnologías, de las prácticas y de las instituciones, el análisis de los códigos. Entre educación presencial y virtual, escuela y medios e interacción e interactividad se suele debatir el campo de la educomunicación. Por otro lado, esta se ha entendido como educación sobre los medios o por los medios, por lo cual siempre se ha tendido a confundir con educación mediática. Este artículo propone la educomunicación como la totalidad de la subjetivación humana, entendida como la asunción de códigos como el lingüístico, y la educación mediática como la asunción de los códigos mediáticos, entendidos no como tecnologías sino como cultura.

\section{Palabras clave (Fuente: tesauro de la Unesco)}

Comunicación; educación; código; cultura; enseñanza multimedia; educomunicación; educación mediática; comunicación educativa. 


\section{Educational Communication, Educommunication, and Media Education: A Research and Educational Proposal from a Culturalist Approach*}

\section{Abstract}

Between face-to-face and virtual education, school and media, and interaction and interactivity, the field of educommunication is often discussed. It has also been regarded as education about media or through media, which is why it is frequently confused with media education. The aim of this article is to review the basic concept of educational communication and its derivatives - educommunication and media education - and then redefine them from a culturalist point of view. From this approach, the categories of the theory of codes are applied to the so-called educommunication, giving rise to three types thereof: oral-ritual, iconic-narrative and alphabetic-argumentative (paradigmatic). In conclusion, educommunication is proposed as the entire human subjectivation, understood as the acquisition of codes - including a particular language - , and media education as the acceptance of media codes, understood not as technologies but as culture. The fundamental contribution here is the expansion of the concept of educommunication by incorporating, in addition to the analysis of technologies, practices and institutions, code analysis.

\section{Keywords (Source: Unesco Thesaurus)}

Communication; education; codes; culture; multimedia instruction; educommunication; media education; educational communication.

\footnotetext{
* This paper is a part of the background and theorical elaboration of the proyect "Educación mediática y nación: entre el púlpito e internet". It is being devolep by the code DPG-437-16 of the Research Center of the Universidad Pedagógica Nacional and coordinated by the author.
} 


\section{Comunicação educativa, educomunicação e educação midiática: uma proposta de pesquisa e formação sob uma abordagem culturalista*}

\section{Resumo}

Entre educação presencial e virtual, escola e meios, e interação e interatividade, costuma-se debater o campo da educomunicação. Esta, por sua vez, tem sido entendida como educação sobre os meios ou pelos meios, razão pela qual sempre se confunde com educação midiática. O objetivo deste artigo é fazer uma revisão do conceito básico de comunicação educativa e suas derivações: educomunicação e educação midiática, para, logo, redefini-los de um ponto de vista culturalista. Com base nessa abordagem, são aplicadas as categorias da teoria dos códigos à chamada educomunicação, que tem como resultado a existência de três tipos de educomunicação: oral-ritual, icônico-narrativa e alfabético-argumentativa (paradigmática). Em conclusão, propõe-se a educomunicação como a totalidade da subjetivação humana, entendida como a aquisição de diferentes códigos, entre eles, a língua particular, e a educação midiática como a assunção dos códigos midiáticos, entendidos não como tecnologias, mas sim como cultura. A contribuição fundamental é a ampliação do conceito educomunicação para incorporar, além da análise das tecnologias, das práticas e das instituições, a análise dos códigos.

\section{Palavras-chave (Fonte: tesauro da Unesco)}

Comunicação; educação; código; cultura; ensino multimídia; educomunicação; educação midiática; comunicação educativa.

* Este artigo faz parte dos antecedentes y e da elaboração teórica do projeto "Educação midiática e nação: entre o púlpito e a internet”, atualmente em andamento sob o código DPG-437-16 do Centro de Pesquisas da Universidad Pedagógica Nacional e coordenado pelo autor. 


\section{La versión institucional}

La versión institucional puede leerse a la luz de tres categorías de igual nivel de abstracción y tres fenómenos de igual grado de institucionalización (tabla 1).

Tabla 1. Versión institucional

\begin{tabular}{|l|l|l|}
\hline \multicolumn{1}{|c|}{ Educación } & \multicolumn{1}{c|}{ Comunicación } & \multicolumn{1}{c|}{ Cultura } \\
\hline \multicolumn{1}{|c|}{ Escuela } & \multicolumn{1}{c|}{ Medios } & Institución artística y patrimonial \\
\hline Educación & Comunicación & Formación \\
\hline Instrucción/inculcación & Información/entretenimiento & Fruición/juicio artísticos \\
\hline Saberes disciplinares & Saberes mediáticos & Saber estético \\
\hline Pedagogía/ciencias de la educación & Mediología/ciencias dela comunicación & Historia del arte/humanidades \\
\hline
\end{tabular}

Fuente: elaboración propia.

En efecto, a cada una de estas esferas o sistemas de la vida social le correspondería una organización institucional que la regiría (Parsons, 1964). Así, por ejemplo, en Colombia, existen tres ministerios distintos, uno para cada una: el Ministerio de Educación Nacional, el Ministerio de Tecnologías de la Información y las Comunicaciones y el Ministerio de Cultura. A cada uno le corresponde, además, regular una institución social que cumple funciones en nombre de la sociedad (Castells, 1999), en este caso, funciones de comunicación pública, es decir, especializada y que se presume autorizada y legítima (Martín, 2009): la escuela, los medios y las infraestructuras tecnológicas y las instituciones artísticas y patrimoniales.

Esto da a entender que la función de cada institución es distinta. A la primera le corresponde, en rigor, educar, como si el sistema escolar tuviera el monopolio de la educación; a la segunda, comunicar, en el sentido de integración física de la población en el territorio a través de las tecnologías de medios y de sus relatos mediáticos; a la tercera, la formación propiamente dicha, en el sentido humanístico, a través de la conservación y la exhibición de lo que se llama el patrimonio y las artes.

Por consiguiente, la actividad de la escuela se reduce a dos procesos: 1) el de instrucción o enseñanza de conocimientos, específicamente de los 
saberes escolares, 2) y el de inculcación o enseñanza de normas éticas, morales y legales y, desde luego, la reproducción del sistema social. La actividad de los medios se centraría, por una parte, en la información, o sea, en la difusión de los relatos no ficcionales; y por otra, en el entretenimiento o difusión de relatos ficcionales. Entretanto, la función de la institución artística sería la de formar a la población en su capacidad de fruición y juicio artístico y patrimonial, es decir, en el gusto legítimo. Cada una de estas acciones institucionales se basa en un tipo de saber distinto; la instrucción y la inculcación, en los saberes disciplinares; la información y el entretenimiento, en los saberes mediáticos y cotidianos; y la formación artística, en los saberes estéticos.

Esta especialización ha dado origen a diferentes campos de estudio, especializados en cada una de las instituciones y en los saberes que transmiten. Así, la pedagogía y las ciencias de la educación son las encargadas de dar cuenta de la educación en su versión escolarizada; las humanidades y la historia del arte son las disciplinas que hacen saber de las instituciones artísticas y la tradición estética; y, cuando se trata de los medios, la identificación de la disciplina que advertiría de ellos sería, convencionalmente, la comunicación. Sin embargo, la comunicación como disciplina sería la comunicología, lo cual desbordaría el estudio de los medios; por eso, existe ya aceptada una parte de la sociología que se ha denominado mediología (Pireddu y Serra, 2014), pero que se puede aceptar como estudios de medios en general o de la comunicación de masas (Wolf, 1996).

Por causa de esta separación institucional, cuando se habla de medios o de comunicación educativa o cultural, lo que se quiere decir es que los medios deberían transmitir tanto los saberes disciplinares como los saberes estéticos, o sea, se pretende que la institución mediática cumpla las funciones de las otras instituciones. Esa es, en parte, la causa del entusiasmo que han despertado los medios como sustitutos o al menos como complementos de la escuela y la institución artística: la creencia de que la comunicación es asunto de medios no de saberes, de culturas. De ahí es que llegamos con entusiasmo a la llamada comunicación educativa. 


\section{La versión tecnologista o comunicación educativa}

La comunicación educativa se suele abordar, por lo menos, en dos grandes líneas de descripción: 1) la de los medios educativos y 2) la de los medios escolares.

\section{Los medios educativos}

Los medios educativos, es decir, las producciones mediáticas que tienen como propósito cumplir los fines de instrucción e inculcación que debe cumplir la escuela (Bourdieu y Passeron, 2001), tienen una larga tradición que se inicia prácticamente con la aparición de los medios de comunicación de masas. Al respecto, es ilustrativa la descripción que hace Sierra (2002) sobre la manera como ya a principios del siglo XX, especialmente en Norteamérica, a la radio se le empiezan a asignar funciones educativas que luego sucumben ante el empuje comercial de la publicidad en los medios privados. Respecto de Colombia, es también esclarecedora la descripción que hace Álvarez (2003) de la forma como desde comienzos del cine y la radio se empieza a vaticinar en el país el fin de la escuela y del libro como institución y medio educativos, respectivamente, así como la presentación que hace Díaz (2005) del papel del cine y la radio en la campaña de cultura aldeana como estrategia educativa llevada a cabo por el Gobierno de la Revolución en Marcha a partir de 1935.

Pero es desde la aparición de la televisión como medio masivo que la idea de reemplazar la escuela por los medios se generaliza, especialmente en el tercer mundo y en América Latina, dada la carencia de cobertura e infraestructura educativas que acusan estos países. Esta época coincide con lo que se ha conocido como la etapa del desarrollismo o de la invención del desarrollo (Escobar, 1998; Martínez, Noguera y Castro, 2003), en la cual el papel de los medios de comunicación es central como instrumento para la difusión de innovaciones en la agricultura y la industria, pero también como sustitutos o complementos de las deficiencias de cobertura de la educación escolar.

Aunque la etapa del desarrollo se inicia en la década de los cincuenta, es a partir de la década de los sesenta que se inicia intencionalmente, por 
parte de los gobiernos y las instituciones educativas, la apuesta por los medios educativos. Desde ahí podemos identificar dos etapas: 1) una que iría desde la década de los sesenta hasta mediados de la década de los ochenta, cuando podemos hablar de la preeminencia de los medios pedagógicos; y 2) otra que va de la segunda mitad de la década de los ochenta hasta la actualidad, cuando podemos hablar de la preeminencia de la informática educativa (tabla 2).

\section{Tabla 2. Componentes de la comunicación educativa}

\begin{tabular}{|c|c|c|c|c|}
\hline \multirow{6}{*}{$\begin{array}{l}\text { Educación- } \\
\text { comunicación }\end{array}$} & \multirow{6}{*}{$\begin{array}{c}\text { Comunicación } \\
\text { educativa }\end{array}$} & \multirow{4}{*}{ Medios educativos } & \multirow[t]{2}{*}{ Medios pedagógicos } & $\begin{array}{l}\text { Enseñanza } \\
\text { audiovisual }\end{array}$ \\
\hline & & & & Ayudas didácticas \\
\hline & & & \multirow{2}{*}{ Informática educativa } & CAT \\
\hline & & & & educación virtual \\
\hline & & \multirow{2}{*}{ Medios escolares } & \multicolumn{2}{|c|}{ Didácticos (gramática) } \\
\hline & & & \multicolumn{2}{|c|}{ Pedagógicos (pragmática) } \\
\hline
\end{tabular}

CAT: computer-assisted teaching.

Fuente: elaboración propia.

Como lo muestra Bullaude (1970), la etapa de los medios pedagógicos se caracteriza por que hay un esfuerzo por sistematizar la experiencia de lo que se llama enseñanza audiovisual y ayudas didácticas, que hace una diferencia entre la tecnología, los productos, la actuación, etc. En ella es característico el énfasis en las posibilidades didácticas y pedagógicas de la imagen, como se puede ver también en los escritos de Jacquinot (1985/1996).

La etapa de la informática educativa, a la vez, tiene dos momentos: el de la última década del siglo XX, centrado en la Computer-assisted teaching (CAT), o sea, en la informática propiamente dicha, y el de la educación virtual, centrado en la telemática (en línea) (Narváez, 2002). La primera se preocupa, fundamentalmente, por el almacenamiento, acceso y procesamiento de la información (Papert, 1993); y la segunda, por la interactividad, el aprendizaje colaborativo, la llamada cibercultura (Lévy, 2007) y la educación virtual (Lévy, 1999), la cual ha devenido más bien un negocio multimedia bajo el nombre de educación telemática (Crovi y Sierra, 2005). 


\section{Los medios escolares}

Los medios escolares admiten dos variantes: 1) una que podemos llamar didáctica, especialmente relacionada con las características comunicativas de los saberes escolares, de la enseñanza, de los textos y materiales didácticos, cuyo centro de atención serían los procesos de codificación de las disciplinas escolares y, por tanto, de la emisión y recepción de la enseñanza y el aprendizaje); y 2) otra que podemos llamar pedagógica, relacionada con los procesos de comunicación en la vida institucional, como una suerte de esfera pública escolar, cuyo centro de atención estaría en los procesos de interacción y el papel de los medios escolares en la convivencia. Pero como toda esfera pública requiere un lenguaje común, entonces tenemos que volver a la codificación (tabla 2).

Como se puede ver, toda la evolución de la comunicación educativa obedece a momentos tecnológicos, aunque coincida también con algunos cambios políticos y sociales y con cambios en la interpretación de la sociedad. Sin embargo, lo que parece determinar la orientación es la socialización de la televisión, el videotape, el computador personal, el internet y ahora el internet 2.0.

\section{Educomunicación}

El cambio de nombre de comunicación educativa a educomunicación no es tan claro conceptualmente como se esperaría. De hecho, este último término designa, por lo menos, tres prácticas distintas, que van desde la militancia política hasta diferentes concepciones epistemológicas (Álvarez, 2009), pasando, como es de esperar, por la tecnología.

\section{Educomunicación como ética y política}

Esta versión de la educomunicación hace parte más bien de lo que se ha llamado educación popular. En tal sentido, es más un movimiento social y una alternativa pedagógica que una propuesta teórica o epistemológica, tal y como lo señala de Oliveira: 
comunitários que, nos meados do século XX, buscavam soluções para seus problemas de comunicação. Uma vez identificada e sistematizada, foi possível à academia propor a prática educomunicativa a diferentes âmbitos sociais, entre os quais 0 midiático e 0 educativo. $(2014 a, \text { p. 136 })^{2}$

Por un lado, se trata de la corriente en boga desde la década de los sesenta que sostiene que los medios audiovisuales son más efectivos para educar al pueblo, dado lo extendido del analfabetismo. Por otro lado, al mismo tiempo que los medios son más efectivos por su facilidad de lectura, también lo son desde el punto de vista de la cobertura por su capacidad de expansión social y geográfica; pero, sobre todo, son una forma de oponerse a los medios dominantes, es decir, oficiales y privados, los cuales serían, por naturaleza, alienantes en cuanto promueven fundamentalmente el consumismo, e ideológicamente reaccionarios en cuanto defensores del statu quo (Álvarez, 2009).

Por este camino se llega a la comunicación alternativa como propuesta de la izquierda marxista y cristiana, en la cual los medios alternativos son, sobre todo, instrumentos ideológicos para la educación y la concientización de las masas populares, tanto campesinas como obreras y barriales (Álvarez, 2009).

Hacia la década de los ochenta esta tendencia sufre una suerte de revisión en cuanto a lo que se consideran las alteridades, y se transforma, bajo el efecto del llamado giro culturalista, en comunicación comunitaria, rótulo que pretendía significar que había más reivindicaciones que las económicas y políticas en los sectores populares, pues se trataba de incluir las reivindicaciones identitarias y culturales que los caracterizaban (Alfaro, 1999).

De ahí en más este campo ha cambiado de nombre y orientación, pasando a llamarse comunicación para el desarrollo para luego transformar-

2 El concepto no nació como consecuencia de la aplicación de resultados de investigaciones a la realidad social, sino que emergió de las prácticas de agentes comunitarios que, a mediados del siglo XX, buscaban soluciones a sus problemas de comunicación. Una vez identificada y sistematizada, fue posible a la academia proponer la práctica educomunicativa a diferentes ámbitos sociales, entre ellos el mediático y el educativo. 
se en comunicación para el cambio social durante la última década (Alfaro, 2016; Barranquero y Ángel, 2015; Cadavid y Gumucio, 2014). Se trata de una propuesta que ya no apunta a la transformación del sistema social en su conjunto, sino al acompañamiento de las comunidades locales o sectoriales en sus procesos de autoorganización para el logro de objetivos reivindicativos dentro del propio sistema. Sin embargo, sigue asumiéndose como un espacio de resistencia a todo lo que se considera hegemónico y como parte de procesos sociales emancipatorios, cualquiera que sea el significado otorgado a este término (Mora, 2014).

\section{Educomunicación como técnica: educación mediatizada}

Por otro lado, existe una tendencia educomunicativa, especialmente en el mundo anglosajón, que sí tiene que ver con los medios, pero que se aleja de la concepción culturalista que expondremos más adelante. Se trata de la llamada media literacy, la cual, traducida como alfabetización mediática, se convierte en un verdadero oxímoron. Como sostienen algunos de sus promotores,

to the domains of reading, writing, and traditional print literacies one could argue that in an era of technological revolution educators must develop robust forms of media literacy, computer literacy, and multimedia literacies, thus cultivating "multiple literacies" in the restructuring of education. Computer and multimedia technologies demand novel skills and competencies and if education is to be relevant to the problems and challenges of contemporary life, engaged teachers must expand the concept of literacy [las cursivas son mías] and develop new curricula and pedagogies. (Kellner y Share, 2005, pp. 369-370) $)^{3}$

Desde nuestro punto de vista, la metáfora es desafortunada, pues, así como no hay múltiples lenguajes, tampoco hay múltiples alfabetizaciones.

3 A los dominios de la lectura, la escritura y las alfabetizaciones tradicionales de la impresión, se podría agregar que en una era de revolución tecnológica los educadores deben desarrollar formas sólidas de alfabetización mediática, informática y multimedia, y así cultivar las alfabetizaciones múltiples en la reestructuración de la educación. Las tecnologías informáticas y multimedia exigen habilidades y competencias novedosas y, para que la educación sea relevante a los problemas y desafíos de la vida contemporánea, los docentes comprometidos deben ampliar el concepto de alfabetización y desarrollar nuevos planes de estudio y nuevas pedagogías. 
La alfabetización se refiere a la cultura alfabética y justamente lo que diferencia la educación escolar de la educación mediática es el predominio de la competencia alfabética frente a la competencia oral e icónica propia de lo mediático. La palabra skill, en la cita, habla más de un adiestramiento en el hacer (como aprender la caligrafía) que de la competencia cultural en la lectura y producción de textos.

En efecto, una de las primeras características de este enfoque es la confusión entre cultura y tecnología, según la cual se nos vende la tecnología como código. Por eso, se habla de cultura digital, virtual y, sobre todo, de cibercultura (Lévy, 2007). Esta es una larguísima tradición que ha tocado hasta los más brillantes analistas como el propio Williams (1992). Para poner un ejemplo cercano, un analista latinoamericano de los más autorizados escribía un artículo titulado "El periodista en la quinta revolución cultural". Las revoluciones culturales de que habla son oralidad, escritura, imprenta, radio y cine y PC (personal computer) (Roncagliolo, 1996, p. 6). Con este criterio, quedan faltando las telecomunicaciones (telégrafo, teléfono, televisión, telemática). Desde luego, la oralidad (el lenguaje), como veremos, no es una revolución cultural sino biológica; la escritura, si se piensa como sistema simbólico y, por consiguiente, también como forma de pensar, sí lo es; pero las demás son tecnologías modernas. Es decir, no son una nueva forma de codificación, sino una forma de reproducción técnica de los mismos códigos (oralidad y escrituras).

Como se explicó en otra parte (Narváez, 2013), existe un lugar común que suele distinguir tres tipos de cultura (Frith, 2000, pp. 204-205): 1) la cultura tradicional, en la cual tanto productores como consumidores tienen la facultad para producir y consumir (Bustamante, 2003), por ello no es necesaria la especialización y es una cultura de la masa para la masa; 2) la cultura mediática, que es básicamente la cultura moderna, en la que los productores necesitan formación especializada (como escritores, compositores o realizadores mediáticos), pero no así los consumidores, y es una cultura de especialistas para la masa; y 3 ) la cultura letrada, en la que tanto productores como consumidores requieren formación especializada, típicamente, aprender a leer y a escribir, además de las competencias 
de pensamiento que exige la lectura, $y$ es una cultura de especialistas para especialistas (tabla 3).

\section{Tabla 3. Periodización de la cultura (Frith, 2000)}

\begin{tabular}{|c|c|c|c|}
\hline Tradicional & \multicolumn{2}{|c|}{ Moderna } & Letrada \\
\hline Oral & \multicolumn{2}{|c|}{ Mediática } & Alfabética \\
\hline Interpersonal & Mediatizada & Oral-icónica & Impresa \\
\hline
\end{tabular}

Fuente: elaboración propia.

¿Cómo se interdefinen esas nociones para poder fungir como categorías? La dicotomía básica es tradicional-moderna, mientras que la cultura letrada aparece como si fuera intemporal. Además, se mezclan categorías incomparables: etapas (tradicional-moderna) con códigos (letrada). Ahora, si la diferencia entre tradicional y moderna radica en la técnica (mediatización), entonces lo opuesto de mediatizada no es tradicional sino interpersonal y, en ese caso, la cultura moderna sería igual a la impresa, que es la mediatización de lo alfabético. Pero si la diferencia es entre oral y audiovisual, entonces la cultura tradicional y la mediática son iguales (oral-icónicas) y ambas se oponen a la letrada alfabética.

En el mismo sentido, podemos mencionar la periodización de Martín (2009), según la cual la socialización cognitiva que llevan a cabo las instituciones encargadas de la comunicación pública habría sido alfabética hasta la década de los sesenta, audiovisual entre esta década y la década de los ochenta, y finalmente ya no más alfabética ni audiovisual sino a través de las tecnologías de la información y de la comunicación (TIC), a partir de la década de los ochenta. Pero, como se puede ver, en las primeras dos etapas habla de códigos, pero en la segunda habla de tecnologías, que no son otros códigos, sino tecnologías de reproducción de los códigos.

Tal vez sea necesario aclarar aquí la diferencia entre la mediación y la mediatización, para lo cual proponemos diferenciar entre comunicación y medios. Así, la comunicación es la condición existencial humana gracias al lenguaje. De él se desprende todo lo que tiene que ver con la sociedad, las 
instituciones morales, el significado, el sentido, el trabajo, la técnica, la ciencia, etc. Los medios, en cambio, son un producto del capitalismo, una suerte de tecnificación de los procesos de comunicación.

Pero el capitalismo ha hecho que reduzcamos la comunicación a los medios, ya sean químicos, electrónicos o digitales; ya sean masivos (broadcasting) o interactivos (narrowcasting). Es decir, ha hecho que pensemos la comunicación y el conocimiento como información, no como significación o cultura. Roncagliolo (1997, pp. 83-104) escribió un artículo pionero que vale la pena rescatar ("Los espacios culturales y su onomástica"), en el que daba cuenta de la red de términos acuñados para nombrar la nueva época y las limitaciones de cada uno. Allí se mencionan nombres como nuevas tecnologías, globalización, internacionalización, transnacionalización, tercera revolución industrial, sociedad posindustrial, autopistas de la información, sociedad de la información, mundialización, tercera revolución cultural, videosfera, gatesismo, etc. Como se ve, todos los nombres tienen que ver con fenómenos del capitalismo.

Al mismo tiempo, ha hecho que pensemos la digitalización de la información en una de sus formas, la digitalización electrónica, no como sistema de señales sino como sistema simbólico. Por tanto, ha hecho que consideremos que existe una cultura digital en vez de una información digitalizada. Por ese mismo camino de la cultura digital nos ha conducido a la llamada educación virtual. Pero estas no son más que metáforas, no muy afortunadas, pues la virtualidad y la condición digital son características del lenguaje, es decir, de lo humano.

En resumen, la digitalización electrónica es una mediatización (tecnología), no una mediación (cultura). Asumir la tecnología (generada), el hacer (skills), sin asumir la cultura científica (generadora), el saber, el pensar, el conocer, es lo que nos reproduce como periféricos. Desde esta perspectiva situamos la educación mediática y la educomunicación dentro del capitalismo cultural, en el cual se reproduce la relación centro-periferia como desarrollo-subdesarrollo, lo cual es avalado por las diferentes clasificaciones mundiales como las pruebas Saber, el ranking de universidades, etc. (Narváez, 2013, 2014b). 


\section{Educomunicación como condición antropológico-cultural}

Nuestra propuesta se basa en lo que consideramos una versión culturalista de la comunicación y la educación, según la cual las culturas son codificaciones del mundo compartidas por los grupos humanos. En ese sentido, la educación y la comunicación son un mismo fenómeno, solo separable analíticamente, consistente en los procesos de transmisión cultural. Entendida como transmisión cultural, la educomunicación es una práctica de transmisión simbólica, y no física ni biológica, lo que quiere decir que involucra principalmente las representaciones, las significaciones y los sentidos. Por tal motivo, se consideran las instituciones y las tecnologías como generadas por los procesos culturales educomunicativos y no al contrario.

Desde esta perspectiva culturalista, hay un punto de partida irreductible: en la sociedad, todo es cultural, la cultura es la verdadera naturaleza humana, por tanto, en la sociedad, la cultura tiene un carácter generativo, no derivado; la cultura es un tipo de saber distinto del genético, que consiste en la capacidad de dar respuestas interpretativas a los estímulos del medio (Eco, 1977/1995). Pero esas respuestas son compartidas a través de sistemas simbólicos, es decir, esos saberes interpretativos permiten al mismo tiempo la comunicación; la educación, por su parte, consiste en la transmisión de toda la herencia no biológica, o sea, simbólica, cultural. Por tanto, cultura, educación y comunicación son la condición humana esencial. Veamos.

Filogenéticamente, el hombre, como especie, tiene cien mil años, en los cuales no hay cambios biológicos (Aitchinson, 1996; Cavalli-Sforza y Cavalli-Sforza, 1999; Kristiansen, 2001). Pero, como toda especie, sobrevive por los individuos; aquí viene la especificidad: ontogenéticamente, el individuo humano nace incompleto. Tiene que esperar a madurar; todavía después de nacido le crece el cerebro y tiene que acondicionar el cuerpo (Skybreak, 2006); por eso, necesita cuidado, para hacerse miembro completo de la especie.

Pero mientras el individuo es un producto biológico, el sujeto es un producto cultural. Mientras el individuo es un miembro de una especie, el 
sujeto es un miembro, no de la especie humana, sino de un grupo humano diferenciado; es un individuo atravesado por una cultura, específicamente por una lengua. Mientras el individuo es el medio por el cual la especie garantiza su supervivencia y su reproducción biológica, el sujeto garantiza la reproducción del grupo o, en sentido amplio, de la etnia, en términos culturales.

En efecto, los dos componentes básicos de la cultura son el mito y el lenguaje (Cassirer, 1993). ¿En qué se educa y cómo se comunica la especie humana? Se educa en valores (la exogamia como principio moral que garantiza la convivencia) y en conocimientos (el trabajo como construcción de instrumentos que garantiza la sobrevivencia), los cuales no existen fuera del lenguaje, de una lengua particular. La educación es la transmisión del mito, entendido como cosmovisión. El mito se transmite por la lengua. Mito y lengua son, pues, la especialidad de la especie humana. Ambos constituyen la cultura y son el nacimiento de la educación y la comunicación.

"El hombre es la única criatura que ha de ser educada”, decía Kant (1803/2003) en una frase lacónica al inicio de su curso de Pedagogía. ¿Cuál es la razón? Precisamente, el lenguaje, el cual le da al animal humano la característica de la etnización, de la que se desprenden la desespeciación y la desespecialización y la neotenia.

La etnización es el paso de la zoología a la sociología (Leroi-Gourhan, 1971). Es el paso del agrupamiento animal al agrupamiento humano. En consecuencia, es un proceso de desespeciación, o sea que la vida humana deja de estar regida genéticamente y comienza a estar regida culturalmente, simbólicamente. Esta ruptura es inédita. Con ella se inicia la tragedia humana: la búsqueda del sentido. Pero, además de la desespeciación, también implica la desespecialización, es decir, no nacer aprendido, tener que aprender todos los comportamientos socialmente útiles y aceptables, que ya no vienen en la sangre sino en la cultura, en la lengua; por eso, necesita la educación.

¿En qué consiste la educación? Kant divide la educación en tres etapas sucesivas: 1) el cuidado, 2) el disciplinamiento y 2) la instrucción. El 
cuidado tiene que ver con la ontogénesis, pero está condicionado culturalmente, mientras el disciplinamiento y la instrucción son típicamente etnogenéticos, culturales. Etnogenéticamente, el sujeto tiene que aprender los valores y comportamientos, es decir, el disciplinamiento. Después, tiene que aprender los conocimientos, es decir, la instrucción. Pero como la cultura es asintótica, nunca encontramos el sentido último, el saber último, siempre estamos aprendiendo, buscando; a eso se le llama neotenia (Virno, 2005), la búsqueda permanente del sentido, el cual no existe, pues si existiera no estaríamos en permanente disputa por imponerlo. Y todo esto gracias al lenguaje. Las palabras no tienen una relación necesaria, ni permanente, ni objetiva, ni física con las cosas. Tampoco hay una respuesta completamente repetible bajo iguales circunstancias, porque no hay dos circunstancias absolutamente iguales en la vida humana. Siempre estamos intercambiando significados $y$, por tanto, dando respuestas interpretativas al medio en que vivimos. A esa capacidad es a lo que Eco (1995) llama comunicación. Es decir, la educación está en la comunicación. O la comunicación es la educación.

Educación y comunicación son, entonces, las características antropológicas básicas. Son los procesos que describen la existencia humana. Cuando hablamos de la vida humana, hablamos de procesos de educación y comunicación. Según lo dicho, si existe algo como la educomunicación, tendría que ser el objeto de la antropología o la etnología, es decir, del estudio de los grupos humanos.

En efecto, la educomunicación que proponemos se entiende como proceso único e indivisible de enculturación, socialización y subjetivación, como asunción de los códigos. En consecuencia, si consideramos el mito como el sistema de conocimientos y el lenguaje como cualquier lengua histórica en uso, esta sería la estructura de la educomunicación en Occidente (tabla 4), donde, además, los procesos de educación se han institucionalizado en la escuela y los de comunicación se han mediatizado a través de las tecnologías. Pero estas últimas son contingentes, son hechos históricos, mientras que la educación y la comunicación son condiciones esenciales y necesarias de la especie humana. 


\section{Tabla 4. Educomunicación como condición cultural}

\begin{tabular}{|c|c|}
\hline Educación & Comunicación \\
\hline Mito & Lenguaje \\
\hline Institucionalización & Mediatización \\
\hline Escuela & Medios y tecnologías de la información \\
\hline
\end{tabular}

Fuente: elaboración propia.

\section{Educomunicación como transmisión cultural}

Pero si la educomunicación es transmisión cultural, entonces la pregunta siguiente es por la cultura. Aquí la cultura se entiende como código, no como producto; es decir, como sistema generador, no como lo generado. Para poner los problemas de la educomunicación en términos culturales, se entiende como un asunto de estructuras comunicativas que oscilan entre dos matrices culturales que permanecen, las cuales no se superan la una a la otra: por un lado, una matriz o "racionalidad expresivo-simbólica popular" opuesta, por otro lado, a una matriz o "racionalidad informativa-instrumental” (Martín-Barbero, 2003, pp. 161-162). En cuanto a la educación, tenemos, por un lado, un tipo de pensamiento llamado narrativo y, por otro, el pensamiento llamado paradigmático (Bruner, 2000). De estas matrices es que se derivan las que en la jerga académica conocemos como culturas populares las primeras y cultura occidental la segunda, denominaciones que obedecen a una nomenclatura extracomunicativa, pero que permiten entendernos en un léxico común.

De las referencias anteriores se puede decir que las culturas son formas de codificar el mundo o el pensamiento sobre el mundo. En tal sentido, la cultura como código se puede sintetizar en una correspondencia sintáctico-semántica como la que aparece en la tabla 5, la cual permite producir al mismo tiempo textos y discursos. ${ }^{4}$

4 "Lo que se llama mensaje es, la mayoría de las veces, un texto, cuyo contenido es un discurso a varios niveles" (Eco, 1995, p. 97), lo cual quiere decir que tiene implicaciones denotativas, connotativas y pragmáticas. 


\section{Tabla 5. Cultura como código}

\begin{tabular}{|c|c|c|c|}
\hline Función semiótica & & & \\
\hline Mensaje & & & \\
\hline Sintáctica & & Semántica & \\
\hline Expresión & & Contenido & \\
\hline Sustancia & Forma & Forma & Sustancia \\
\hline $\begin{array}{c}\text { Texto } \\
\text { Analógico/digital }\end{array}$ & $\begin{array}{l}\text { Figurativa/abstracta } \\
\text { Icónica/alfabética } \\
\text { Analógica/digital }\end{array}$ & $\begin{array}{c}\text { Figurativa/abstracta } \\
\text { Narrativa/argumentativa } \\
\text { (paradigmática) } \\
\text { Sintética/analítica }\end{array}$ & $\begin{array}{c}\text { Discurso } \\
\text { Situacional/trascendental }\end{array}$ \\
\hline
\end{tabular}

Fuente: elaboración propia.

Entre el texto y el discurso existe la misma distancia que entre la fonética y la semántica. Para que los sonidos de la lengua tengan algún significado tienen que pasar por las reglas de la fonología, la morfología y la sintaxis de la lengua. Entre el texto y el discurso median, entonces, las formas, tanto de expresión como de contenido, esto es, las reglas que permiten dar significado a esa expresión material llamada texto. Las formas son, por decirlo así, el secreto de la codificación y son, por tanto, el núcleo de la cultura, puesto que son la parte no evidente pero generativa de la comunicación, mientras que los textos y los discursos son evidentes pero generados por las formas.

Desde este punto de vista, las culturas se definen y se diferencian según las siguientes características: 1) por sus códigos, esto es, si son figurativas o abstractas; 2) por su discursividad, o sea, si son narrativas o argumentativas; y 3 ) por su forma de aprendizaje y transmisión, esto es, si son textualizadas o gramaticalizadas, es decir, si los textos preceden a las reglas o si las reglas preceden al texto (Eco, 1977/1995, pp. 97-99).

Estas características tienen implicaciones en su reproducción y transmisión, por cuanto determinan si predomina el aprendizaje o la enseñanza o el aprendizaje espontáneo o el aprendizaje por estudio y reflexión (tabla 6). 


\section{Tabla 6. Rasgos predominantes de las tradiciones culturales}

\begin{tabular}{|l|l|}
\hline \multicolumn{1}{c|}{\begin{tabular}{c}
\multicolumn{1}{c|}{$\begin{array}{c}\text { Tradición } \\
\text { oral-icónica narrativa } \\
\text { (popular) }\end{array}$} \\
Figurativa
\end{tabular}} & \multicolumn{1}{c|}{$\begin{array}{c}\text { Tradición } \\
\text { alfabética paradigmática } \\
\text { (ilustrada) }\end{array}$} \\
\hline Narrativa & Abstracta \\
\hline Sintética & Argumentativa \\
\hline Alegorías & Analítica \\
\hline Textualizada (mensajes) & Categorías \\
\hline Se aprende & Gramaticalizada (reglas) \\
\hline Se aprende por patrones & Se enseña \\
\hline Se aprende asistemáticamente & Se aprende por algoritmos \\
\hline
\end{tabular}

Fuente: elaboración propia.

La mainstream de la educomunicación se basa en la pretensión de transponer una cultura en otra. Sin embargo, tomando como base estas características, las culturas son comparables pero no conmensurables en el sentido kuhniano, es decir, no hay equivalentes sintáctico-semánticos entre unas y otras, así como no hay categorías equivalentes entre un paradigma y otro (Kuhn, 1987/1994) y, por tanto, cualquier forma de transposición resulta espuria.

Esto tiene consecuencias en la educomunicación más profundas de lo que queremos admitir. En efecto, ello significa que una cultura abstracta no se puede aprender ni se puede transmitir por medios figurativos; una cultura argumentativa no se puede aprender ni se puede transmitir de manera narrativa; una cultura gramaticalizada no se puede aprender ni se puede transmitir de manera textualizada; la transmisión de una cultura gramaticalizada no puede prescindir de la enseñanza; y finalmente, el aprendizaje de una cultura gramaticalizada no puede prescindir del estudio y la reflexión.

En consecuencia, la educomunicación es diferenciada según se trate de una cultura u otra. Esta diferencia se expresa en dos tipos de educación: la educación escolar y la educación mediática, que viene a ser la mediatización de la educación cotidiana, pues, antes de que hubiera escuela y medios modernos, hubo educación. 


\section{Educación mediática}

Este tampoco es un término nuevo, pero, si se trata de una versión culturalista, hay que resignificarlo. 'O tema da educação 'sobre' e 'com' a mídia tem sido objeto de inúmeros congressos nos últimos anos" (El tema de la educación "sobre" y "con" los medios ha sido objeto de innumerables congresos en los últimos años) (De Oliveira, 2014a, p. 15). A nuestro modo de ver, son dos campos distintos. La educación "sobre" medios sería una "educação para uma recepção ativa e crítica das mensagens midiáticas" (educación para una recepción activa y crítica de los mensajes mediáticos) (p. 16), la cual, agregamos, se puede hacer desde la ética o desde la gramática. En cambio, la educación “con” medios se asemeja más a la media literacy, como también lo entiende el autor.

Entendiendo la educomunicación como un fenómeno indivisible que solo se puede separar analíticamente, consistente en la apropiación de determinados códigos sintáctico-semánticos, la educomunicación se puede observar en manifestaciones como la educación mediática, la educación escolar y educación cotidiana. Sin embargo, desde el punto de vista de los códigos, se realiza como educación icónico-narrativa, educación alfabético-argumentativa y educación oral-ritual, respectivamente (tabla 7).

\section{Tabla 7. Educomunicación como institucionalización y codificación}

\begin{tabular}{|c|c|}
\hline Institucionalización & Codificación \\
\hline Educación cotidiana & Oral-ritual \\
\hline Educación mediática & Icónico-narrativa \\
\hline Educación escolar & Alfabético-argumentativa \\
\hline
\end{tabular}

Fuente: elaboración propia.

Aquí el interés se desplaza, en el primer caso, de la educación como escolarización a la educación como alfabetización; en el segundo, de los medios educativos, como mediatización de la enseñanza, a la educación mediática como mediación, o sea, como cultura icónico-narrativa, no como medios tecnológicos; en el tercer caso, de los valores familiares y sociales, a la tradición oral y sus realizaciones en rituales cotidianos, no mediatizados ni institucionalizados. 
Para tal efecto, aparte de la teoría de las matrices culturales que explica la mediación en la recepción (Martín-Barbero, 2003), partimos de dos grandes enfoques teóricos: por un lado, la teoría de la mediación, según la cual los medios llevan a cabo dos tipos de mediación: estructural y cognitiva, la ritualización y la mitificación, respectivamente (Martín, 1997). Pero, para identificar estos procesos, recurrimos a la teoría de los códigos (Eco, 1995), la cual nos permite identificar la ritualización en las sustancias expresivas (textos) y las formas de expresión y contenido y la mitificación en las sustancias de contenido (discursos) (tabla 8).

\section{Tabla 8. Componentes de la educación mediática}

\begin{tabular}{|l|c|c|c|}
\hline \multirow{2}{*}{ Educación-comunicación } & \multirow{2}{*}{ educación mediática } & La mediación de la emisión (medios) & Cognitiva (mito) \\
\cline { 3 - 4 } & & $\begin{array}{c}\text { La mediación en la recepción } \\
\text { (audiencia) }\end{array}$ & Matriz cultural ilustrada \\
\cline { 3 - 4 } & & Matriz cultural popular \\
\hline
\end{tabular}

Fuente: elaboración propia.

Para entender los medios, no como mediatización sino como mediación, hace falta centrarse en las características comunicacionales, es decir, en los códigos y las narrativas de la oferta mediática, y no solo en sus contenidos o en la tecnología. En este caso, la categoría de primer nivel es la cultura, mientras que educación escolar y educación mediática son del segundo y las tecnologías pasan a un sexto nivel de abstracción (tabla 9).

\section{Tabla 9. Educomunicación}

\begin{tabular}{|c|c|}
\hline Educación escolar & Educación mediática \\
\hline Tradición alfabética & Tradición oral-icónica \\
\hline Tradición argumentativa & Tradición narrativa \\
\hline Cultura escolar & Cultura mediática \\
\hline Escuela & Medios y tecnologías de la información \\
\hline Educación presencial & Educación virtual \\
\hline Pedagogía & Mediología \\
\hline Educomunicología & Educomunicología \\
\hline
\end{tabular}

Fuente: elaboración propia. 
Ahora, si de lo que se trata es de dar cuenta de lo que se llama cultura escolar y cultura mediática como culturas, educativa la primera y de infoentretenimiento la segunda, reiteramos que esta clasificación no corresponde al carácter intrínseco de las culturas, que no son excluyentes, que dependen de las competencias del usuario, y por tanto el hecho de que alguien se entretenga, se informe o se divierta con uno u otro producto cultural es un asunto de la capacidad de los sujetos para consumir determinados productos, quienes, en todo caso, se educan, por cuanto se enculturizan.

\section{A manera de síntesis}

En los planes de desarrollo de la educación, se concibe la modernización de esta en varios sentidos (Roncancio, 2010): 1) como la evolución de los medios analógicos a los medios digitales electrónicos; 2) como la evolución de la palabra oral y alfabética a la imagen (que incluye la imagen de la palabra que es el audiovisual); 3) como el paso de la argumentación al storytelling, es decir, a la narrativa; y 4) como el paso de los contenidos trascendentales a los situacionales (de la disciplina al contexto, como dicen algunos).

Esta pretensión comporta varios equívocos: la primera evolución, a medios digitales e interactivos, constituye una posibilidad tecnológica, un cambio en las sustancias expresivas que son, en rigor, ajenas a la comunicación; los dos cambios siguientes son el paso a las formas de la cultura popular, es decir, a la cultura que no produce ni reproduce el conocimiento propio de las disciplinas escolares, el cual está codificado alfabética y argumentativamente. La primera característica corresponde a una educación mediatizada, de la cual ya hablamos. Las últimas serían las características de la educación mediática propiamente dicha (icónica, narrativa y situacional), que la diferenciarían nítidamente de la educación escolar.

Pero el paso de los códigos abstractos a los figurativos y del pensamiento paradigmático al narrativo lo podríamos sintetizar como el paso inicial de la transformación de la interacción cara a cara en interactividad (Bruner, 2000). Sería algo así como la mediatización de la educación cotidiana (tabla 7). 
Como se ve, el camino de la comunicación educativa a la educación mediática como parte de esa tradición llamada educomunicación no es fácil de recorrer, teniendo en cuenta la confusión de intereses que rodean la construcción de este campo. Como vimos, vamos de la institución (prácticas oficiales de educación a través de los medios) a las prácticas contestatarias o de mera resistencia; de estas a las tecnologías y, finalmente, de las tecnologías a la educomunicación como proceso de transmisión simbólica y de reproducción cultural, la cual incluye las dos grandes tradiciones culturales, la alfabética y la oral-icónica, esta última convertida en cultura mediática, lo cual nos permite pensar la educación mediática, no como destreza técnica, sino como competencia cultural.

Con esta esquematización, se pretende abrir el debate sobre si los problemas, aparentemente de investigación, que se preguntan por las relaciones entre escuela y TIC o entre educación presencial y virtual (mediatización) son en realidad preguntas pertinentes a este campo o si son solo preguntas de un orden distinto del de la investigación en educación-comunicación, pues estos últimos tendrían que ver con las características de la codificación y de la transmisión cultural (mediación) y no, como ocurre mayoritariamente hoy, con la técnica (mediatización) ni con la política (las instituciones y los grupos) ni con la economía (los negocios de las empresas de tecnología, telecomunicaciones y contenidos).

\section{Referencias}

Aitchison, J. (1996). The seeds of speech: Language origin and evolution. Cambridge: Cambridge University Press.

Alfaro Moreno, R. M. (1999). Culturas populares y comunicación participativa: en la ruta de las definiciones. Escribanía: comunicación, cultura, región, 3, 3-14.

Alfaro Moreno, R. M. (2016). ¿Es posible generar desarrollo desde la comunicación? Revista Latinoamericana de Ciencias de la Comunicación, 12(23), 16-24. Recuperado https://www.alaic.org/revista/ index.php/alaic/article/view/745 
Álvarez Gallego, A. (2003). Los medios de comunicación y la sociedad educadora: :ya no es necesaria la escuela? Bogotá: Magisterio.

Álvarez Jaramillo, L. E. (2009). Estilos de conocimiento en los estudios de la comunicación mediática en Colombia: años 1962 a 1990. Popayán: Universidad del Cauca.

Ángel Botero, A. y Obregón, R. (2011). Un análisis crítico de las perspectivas de diálogo en la literatura sobre comunicación para el desarrollo y cambio social: abordajes y desafíos. Signo y Pensamiento, 58, 190-205. Recuperado de http://www.scielo.org.co/scielo. php?script=sci_abstract\&pid=S0120-48232011000100012

Barranquero Carretero, A. y Ángel Botero, A. (2015). La producción académica sobre comunicación, desarrollo y cambio social en las revistas científicas de América Latina. Signo y Pensamiento, 67, 30-58. Recuperado de http://www.scielo.org.co/scielo.php?pid=S0120$48232015000200002 \&$ script $=$ sci_abstract\&tlng $=$ es

Bourdieu, P. y Passeron, J-C. (2001). La reproducción: elementos para una teoría del sistema de enseñanza. Barcelona: Editorial Popular.

Bruner, J. (2000). La educación, puerta de la cultura. Madrid: Visor.

Bullaude, J. (1970). Enseñanza audiovisual y comunicación: teoría y crítica (2. a ed.). Buenos Aires: Librería del Colegio.

Bustamante, G. (2003). El concepto de competencia III: un caso de recontextualización. Bogotá: Sociedad Colombiana de Pedagogía.

Cadavid Bringe, A. y Gumucio Dagron, A. (eds.) (2014). Pensar desde la experiencia: comunicación participativa en el cambio social. Bogotá: Universidad Minuto de Dios.

Cassirer,E. (1993).Antropología filosófica. Bogotá: Fondo de Cultura Económica. 
Castells, M. (1999). La era de la información (vol. 3). México: Siglo XXI.

Cavalli-Sforza, L. y Cavalli-Sforza, F. (1999). ¿Quiénes somos? Historia de la diversidad humana. Madrid: Crítica.

Crovi, D. y Sierra, F. (2005). Sociedad de la información y educación telemática: economía, política y lógicas de socialización del conocimiento. En C. Bolaño, G. Mastrini y F. Sierra (eds.), Economía política, comunicación y conocimiento: una perspectiva crítica latinoamericana (pp. 149-180). Buenos Aires: La Crujía.

Díaz, C. J. (2005). El pueblo, de sujeto dado a sujeto político por construir: el caso de la campaña de cultura Aldeana en Colombia (1934-1936). Bogotá: Universidad Pedagógica Nacional.

Eco, U. (1977/1995). Tratado de semiótica general (5. a ed.). Barcelona: Lumen.

Escobar, A. (1998). La invención del tercer mundo: construcción y reconstrucción del desarrollo. Bogotá: Norma.

Frith, S. (2000). Entertainment. En J. Curran y M. Gurevitch, Mass media and society. Londres: Arnold.

Hall, S. (2008). Richard Hoggart, The Uses of Literacy and the cultural turn. En S. Owen (ed.), Richard Hoggart and cultural studies (pp. 20-32). Londres: Palgrave Macmillan.

Jacquinot, G. (1985/1996). La escuela frente a las pantallas. Buenos Aires: Aique.

Kant, I. (1803/2003). Pedagogía. Madrid: Akal.

Kellner, D. y Share, J. (2005). Toward critical media literacy: Core concepts, debates, organizations, and policy. Discourse: Studies in the Cultural Politics of Education, 26(3), 369-386. DOI: https://doi. org/10.1080/01596300500200169 
Koltay, T. (2011). The media and the literacies: Media literacy, information literacy, digital literacy. Media, Culture \& Society, 33(2), 211-221. DOI: https://doi.org/10.1177/0163443710393382

Kristiansen, K. (2001). Europa antes de la historia. Barcelona: Península.

Kuhn, T. (1987/1994).¿Qué son las revoluciones científicas? Barcelona: Altaya.

Leroi-Gourhan, A. (1971). El gesto y la palabra: técnica y lenguaje. Caracas: Universidad Central de Venezuela.

Lévy, P. (1999). ¿Qué es lo virtual? Barcelona: Paidós.

Lévy, P. (2007). Cibercultura: la cultura de la sociedad digital. México: Anthropos.

López Rojas, M. del M. (2014). La exposición de museo: un lugar común. Revista Nexus Comunicación, 14, 48-71. DOI: https://doi. org/10.25100/nc.v0i14.746

Martín Serrano, M. (1997). La mediación de los medios. En J. Martín-Barbero y A. Silva (comps.), Proyectar la comunicación. Bogotá: Universidad Nacional de Colombia.

Martín Serrano, M. (2009). La producción social de comunicación. Madrid: Alianza.

Martín-Barbero, J. (1987/1991). De los medios a las mediaciones. México: Gustavo Gili.

Martín-Barbero, J. (2003). Oficio de cartógrafo: travesías latinoamericanas de la comunicación en la cultura. Bogotá: Fondo de Cultura Económica.

Martínez Boom, A., Noguera, C. y Castro, J. (2003). Currículo y modernización: cuatro décadas de educación en Colombia. Bogotá: Magisterio. 
Mattelart, A. y Schmucler, H. (1983). América Latina en la encrucijada telemática. Barcelona: Paidós.

Media Literacy Project (s. f.). Introduction to media literacy. Recuperado de http://medialiteracyproject.org/introduction-media-literacy/

Mora, A. I. (comp.) (2014). Comunicación educación un campo de resistencias. Bogotá: Universidad Minuto de Dios.

Narváez Montoya, A. (2002). Puentes tecnológicos, abismos sociales. Manizales: Universidad de Manizales.

Narváez Montoya, A. (2013). Educación y comunicación: del capitalismo informacional al capitalismo cultural. Bogotá: Universidad Pedagógica Nacional.

Narváez Montoya, A. (2014a). Ciencias sociales y giro culturalista: promesa pendiente. Nómadas, 41, 97-113. Recuperado de http:// nomadas.ucentral.edu.co/index.php/inicio/55-trayectos-y-posibilidades-en-ciencias-sociales-nomadas-41/800-ciencias-sociales-y-giro-culturalista-promesa-pendiente

Narváez Montoya, A. (2014b). ¿Sociedad digital o capitalismo cultural? El informacionalismo como utopía neoliberal. Eptic online: revista electronica internacional de economia política da informaçao, da comuniçao e da cultura, 16(3), 116-134. Recuperado de https:// seer.ufs.br/index.php/eptic/article/view/116/

Narváez Montoya, A. (2015). educomunicación y TIC: ni tecnologías de la comunicación ni para la educación. En Á. Camargo Uribe (ed.), Educación y tecnologías de la información y la comunicación (pp. 199218). Bogotá: Universidad Pedagógica Nacional.

Papert, S. (1993). The children's machine: Rethinking school in the age of the computer. Nueva York: Basic Books. 
Parsons, T. (1964). The social system. Nueva York: The Free Press.

Pireddu, M. y Serra, M. (eds.) (2014). Mediología: cultura, tecnología y comunicación. Barcelona: Gedisa.

Roncagliolo, R. (1996). El periodista en la quinta revolución cultural. Chasqui: Revista Latinoamericana de Comunicación, 54. Recuperado de https://revistachasqui.org/index.php/chasqui/article/view/1020

Roncagliolo, R. (1997). Los espacios culturales y su onomástica. Diálogos de la comunicación, 50, 83-104. Recuperado de http://dialogosfelafacs.net/wp-content/uploads/2015/50/Los_espacios.pdf

Roncagliolo, R. (1998). Las industrias culturales en la videosfera latinoamericana. Media-ciones, 1, 47-54. Recuperado de http://www.scielo.org.bo/scielo.php?script=sci_arttext\&pid $=$ S1815-02762002000200013

Roncancio, G. (2010). Políticas de comunicación educativa en colombia 19952010 (Tesis de maestría, Universidad Pedagógica Nacional de Colombia, Colombia).

Sierra Caballero, F. (2002). Educación, comunicación y desarrollo: apuntes para una historia de la comunicación educativa. Sevilla: Comunicación Social, Ediciones y Publicaciones.

Skybreak, A. (2006). La ciencia de la evolución y el mito del creacionismo. Bogotá: Tadrui.

Oliveira Soares, I. de (2014a). Construção de roteiros de pesquisa a partir dos livros da coleção Educomunicação (Editora Paulinas). Comunicação \& Educação, 19(2), 135-142.

Oliveira Soares, I. de (2014b). Educomunicação e Educação Midiática: vertentes históricas de aproximação entre comunicação e educação. 
Comunicação \& Educação, 19(2), 15-26. Recuperado de http:// www.revistas.usp.br/comueduc/article/view/72037

Virno, P. (2005). Cuando el verbo se hace carne: lenguaje y naturaleza humana. Madrid: Traficantes de Sueños.

Williams, R. (ed.) (1992). Historia de la comunicación. Barcelona: Bosch.

Wolf, M. (1996). La investigación de la comunicación de masas. Barcelona: Paidós. 